\title{
The Decay Rate of Ocean Swell Observed by Altimeter
}

\author{
I. R. YOUNG \\ Research School of Earth Sciences, Australian National University, Canberra, Australian Capital Territory, Australia \\ A. V. BABANIN AND S. ZIEGER \\ Faculty of Engineering and Industrial Sciences, Swinburne University of Technology, Melbourne, Victoria, Australia
}

(Manuscript received 22 April 2013, in final form 19 July 2013)

\begin{abstract}
Altimeter data from transects across the Southern Ocean are analyzed to determine the decay of oceanic swell. The resulting decay rate is shown to be proportional to wavenumber squared and swell amplitude cubed. Such a decay relationship is consistent with turbulent interaction with the background, either in the air or water. The present data cannot distinguish between these two cases. The results are consistent with the limited previous studies and present a source term suitable for use in wave prediction models.
\end{abstract}

\section{Introduction}

Ocean swell is typically characterized as waves that have propagated away from their generation region and are no longer receiving active energy input from the local wind. As such, they can be represented by $U_{10} / C<1$, where $U_{10}$ is the wind speed measured at a reference height of $10 \mathrm{~m}$, and $C$ is the phase speed of the waves. A number of studies (Barstow 1996; Young 1999; Chen et al. 2002; Gulev et al. 2003; Sterl and Caires 2005; Gulev and Grigorieva 2006; Semedo et al. 2011) have shown that swell is ubiquitous in the open ocean. Semedo et al. (2011) estimate that averaged over the full globe, $75 \%$ of the time wave conditions are dominated by swell. Excluding the intense wind belts at high latitudes in both hemispheres, this figure increases to more than $85 \%$. Young (1999) and Chen et al. (2002) have shown that the vast majority of this swell is generated at these high latitudes and propagates across the oceanic basins. Indeed, within the Pacific Ocean, clear "swell fronts" can be seen migrating north-south across the basin with the seasons. The position of the front is determined by the relative strength of winds at high latitudes in each hemisphere.

Corresponding author address: I. R. Young, Research School of Earth Sciences, Australian National University, Canberra, ACT 0200, Australia.

E-mail: ir.young@anu.edu.au
The fact that swell energy can propagate such large distances indicates that the decay rate of such waves is small. There have, however, been very few studies of the propagation and decay of swell. Laboratory studies by Young and Sobey (1985), Donelan et al. (1997), and Babanin and Haus (2009) have provided some insight into potential mechanisms for swell decay and studies by Hogstrom et al. (2009) and Semedo et al. (2009) have investigated the interaction of swell with the atmosphere. However, only two field experiments (3 papers) have investigated the decay rates of swell (Snodgrass et al. 1966; Collard et al. 2009; Ardhuin et al. 2009). In both of these cases, the authors have attempted to track individual swell packets along great circle paths. In the case of Snodgrass et al. (1966) in situ instruments were used, whereas Collard et al. (2009) and Ardhuin et al. (2009) used satellite-borne Synthetic Aperture Radar (SAR). In both cases, the challenges of tracking the individual packs radiating out from storm events means that the respective datasets are not extensive, with limited point measurements along the propagation path.

The present study attempts to address these issues by considering cases where Southern Ocean swell propagates along the great circle path traced out by descending (from north to south) altimeter passes. By considering cases where the wind speed, as measured by the altimeter is low, it can be assumed that there is little atmospheric input and, as the resulting spectrum will be narrow banded (swell), the influence of nonlinear wave-wave 
interactions can be neglected. Thus, for deep water conditions, the only active source term will be swell decay. The swell energy is tracked over a distance of approximately $1400 \mathrm{~km}$. Although this is a significantly shorter distance than for either Snodgrass et al. (1966) or Collard et al. (2009), the altimeter provides observations at approximately $14-\mathrm{km}$ intervals along the great circle. This enables a detailed study of the swell decay as a function of propagation distance. In addition, more than 200 individual cases were identified within the database, providing much more data than from previous studies.

The arrangement of the paper is as follows. Section 2 provides a summary of previous studies of swell decay. This is followed in section 3 by a description of the altimeter database and the selection of data for the present study. Section 4 provides a description of the propagation model used to describe the decay of the swell along the great circle path traced out by the altimeter. Section 5 describes the results from application of this model, a discussion of the results and comparisons with previous studies, as well as a discussion of application of the results in a third-generation wave model. Conclusions are presented in section 6 .

\section{Previous studies of swell decay}

The best known study of swell decay is that reported by Snodgrass et al. (1966), in which a series of in situ instruments were arranged along a great circle path across the Pacific Ocean. Swell events generated in the Southern Ocean were tracked along this path. To interpret these and other results, the terminology proposed by Collard et al. (2009) is adopted. The decay of the energy spectrum $F(f, \theta)$ is represented as

$$
F(f, \theta)=F_{0}\left(f, \theta_{0}\right) \exp \left(\int_{t_{0}}^{t}-\mu C_{g} d t\right),
$$

where the subscript " 0 " refers to conditions at the initial location along the great circle, $C_{g}=g / 2 \omega$ is the deep water group velocity, and $\omega=2 \pi f$ is the wave frequency. The decay rate $\mu\left(\mathrm{m}^{-1}\right)$ and its inverse $L_{e}=1 / \mu$ is the $e$ folding length. For constant $C_{g}$ and $\mu$, (1) becomes $F(f, \theta)=F_{0}\left(f, \theta_{0}\right) \exp (-\mu \Delta x)$ and $\Delta x=C_{g} \Delta t$ is the propagation distance along the great circle. It should be pointed out that the assumption of a constant decay rate $\mu$ has no basis in wave physics and, as will be shown later, is not consistent with the observational data. Nevertheless, such a coefficient has been used in the literature and provides an order of magnitude estimate of swell decay rates.

For swell frequencies higher than $f=0.077 \mathrm{~Hz}$ ( $T=13 \mathrm{~s}$ ), Snodgrass et al. (1966) found $\mu \approx 2 \times 10^{-7} \mathrm{~m}^{-1}$ (Collard et al. 2009). For lower frequencies, the decay rate was reduced (i.e., smaller $\mu$, possibly zero) but they could not determine a value within the background noise.

Collard et al. (2009) considered SAR images of wave energy propagating away from storms to determine the decay rate of the swell. Considering cases from the North Pacific Ocean, they found that decay rates for $f=0.067 \mathrm{~Hz}$ $(T=15 \mathrm{~s})$ were approximately $3.7 \times 10^{-7} \mathrm{~m}^{-1}$, with $H_{0} \approx 4.4 \mathrm{~m} \quad\left[H_{0}\right.$ - swell height corresponding to $\mathrm{H}_{0}^{2} / 16=$ $\left.\int F_{0}\left(f, \theta_{0}\right) d f d \theta_{0}\right]$. Throughout this paper, three different representations of the wave height are used. The significant wave height $H_{s}=4 \sqrt{\int F d f d \theta}$ follows the usual definition for waves within a full spectrum. For the case of a narrowbanded (single frequency or monochromatic) spectrum, the waves become swell with a height equal to $H_{\mathrm{ss}}=2 a$, where $a$ is the swell amplitude. When considering swell propagating along a great circle path, the initial value of the swell height can be represented by $H_{0}$, with the swell height at any point along the great circle described by $H_{\mathrm{ss}}$.

Ardhuin et al. (2009), using the same data as Collard et al. (2009), speculated that the swell decay could be the result of shear stress modulations caused by the swell orbital velocities in the atmosphere. In this case, the dissipation will depend on the surface roughness and a critical Reynolds number $R_{e}=4 u_{\mathrm{orb}} a_{\mathrm{orb}} / \nu_{a}$, which defines the transition from laminar to turbulent flow. In the above, $u_{\text {orb }}$ and $a_{\text {orb }}$ are the orbital velocity and amplitude, respectively, in the air at the surface, and $\nu_{a}$ is the viscosity of the air. For $R_{e}<10^{5}$, the flow is laminar and the dissipation coefficient is given by (Dore 1978)

$$
\mu_{v}=2 \frac{\rho_{a}}{\rho_{w} g C_{g}}\left(\frac{2 \pi}{T}\right)^{5 / 2} \sqrt{2 \nu_{a}}
$$

where the subscript for $\mu$ signifies the laminar case; $\rho_{a}$ and $\rho_{w}$ are the air and water densities, respectively; $T$ is the swell period; and $\nu_{a}=1.4 \times 10^{-5} \mathrm{~m}^{2} \mathrm{~s}^{-1}$ is the viscosity of the air. For $T=15 \mathrm{~s}$, (2) yields $\mu_{\nu} \approx 1 \times 10^{-8} \mathrm{~m}^{-1}$, an order of magnitude smaller than the observed decay rates.

For larger values of the Reynolds number, the flow will become turbulent and Ardhuin et al. (2009) indicate that the dissipation rate can be represented by

$$
\beta=-\frac{d E_{s} / d t}{E_{s}}=C_{g} \mu=16 \frac{\rho_{a} 4 \pi^{2}}{\rho_{w} g T^{2}} f_{e} u_{\mathrm{orb}},
$$

where $f_{e}$ is a swell dissipation factor, which for smooth surfaces is on the order of $0.002-0.008$ (Jensen et al. 1989). In (3), $E_{s}=\int F d f d \theta=H_{\mathrm{ss}}^{2} / 16$ is the total swell energy and a lower bound estimate of the orbital velocity can be given by $u_{\text {orb }}=2 \sqrt{E_{s}} 2 \pi / T$. Direct evaluation of (3) with the above values of $f_{e}$ and $u_{\text {orb }}$ yield a $\mu$ an order of magnitude smaller than the observed values. 
However, in field conditions, the values of $f_{e}$ and $u_{\text {orb }}$ may vary significantly from these assumed values. It should be noted that the factor 16 in (3) was missing in Ardhuin et al. (2009), a typographical error (F. Ardhuin 2013, personal communication).

Babanin $(2011,2012)$ proposed essentially the same mechanism as Ardhuin et al. (2009), but on the water side of the interface. That is, above a critical Reynolds number the orbital motion of the swell would induce turbulent flow in the water, which would dissipate energy. Based on observations of the wave-mixed upperocean layer and laboratory experiments, Babanin (2006) was able to show that the critical Reynolds numbers was given by $R_{e_{\text {wave }}}=a u_{\mathrm{orb}} / \nu_{w} \approx 3000$, where $a$ is the amplitude of the swell, $u_{\text {orb }}$ is the orbital velocity in the water, and $\nu_{w}$ is the viscosity of water. If the three-dimensional turbulence already exists, and the ocean is always turbulent, such turbulence is unstable to wave stretching and takes energy from the mean wave orbital motion (e.g., Benilov 2012). Babanin and Haus (2009) found that the dissipation rate of monochromatic, mechanically generated waves is proportional to $a^{3}$. Babanin (2012) used these experimental results, together with the derivation proposed by Babanin (2011), to develop a decay rate relationship of the form

$$
\frac{d a^{2}(x)}{d x}=-\frac{4}{3} b_{1} k^{2} a^{3} .
$$

Noting that $E_{s}$ is proportional to $a^{2}$, that $C_{g} d a^{2} / d x=$ $d a^{2} / d t$, and using the deep water results $\omega^{2}=g k$ and $C_{g}=g / 2 \omega$, (3) can be rewritten as

$$
\frac{d a^{2}(x)}{d x}=-32 f_{e} \frac{\rho_{a}}{\rho_{w}} k^{2} a^{3} .
$$

Comparing (5) and (4), it can be shown that the formulations of Ardhuin et al. (2009) and Babanin (2012) are, not surprisingly, functionally equivalent with

$$
b_{1}=24 f_{e} \rho_{a} / \rho_{w} .
$$

This same functional dependence was originally derived by Bowden (1950) based on different arguments, that is, a presumed analogy between viscous and turbulent viscosity in the water. He argued that both should affect the dissipation of ocean swell. For molecular viscosity, the rate of dissipation can be derived analytically (e.g., Lamb 1932). Bowden adopted the same formulation for the turbulence case, by replacing the coefficient of water molecular viscosity with a coefficient for eddy viscosity. The latter was argued to be a function of wavelength, wave amplitude and period and was obtained by means of dimensional argument.
Babanin (2012) showed that (4) can be solved analytically. Noting that $a=a_{0}$ for $x=0,(4)$ can be solved to yield

$$
\frac{a_{0}}{a(x)}=1+\frac{2}{3} b_{1} k^{2} a_{0} x
$$

Because $a=H_{\mathrm{ss}} / 2$ and $a_{0}=H_{0} / 2$, where $H_{\mathrm{ss}}$ is the swell height, (7) can be expressed in nondimensional form as

$$
\frac{H_{0}}{H_{\mathrm{ss}}}=1+\frac{1}{3} b_{1}\left(H_{0} k^{2} x\right)
$$

and $x$ is the propagation distance from a point where $H_{\mathrm{ss}}=H_{0}$.

Based on laboratory data, Babanin (2012) proposed that $b_{1} \approx 0.004$, this value probably representing an upper bound. Babanin (2012) applied the result to the data of Collard et al. (2009) yielding a reported lower value of $b_{1}=0.002$. There was, however, an error in the text of Babanin (2012) and the actual comparison value is even lower (i.e., $b_{1}=0.001$ ). A value of $b_{1}=0.001$ corresponds to $f_{e}=0.042$, which is larger than the range specified by Ardhuin et al. (2009) of $-0.001<f_{e}<0.019$.

As the functional forms of (3) and (4) are the same, data that measure the rate of decay of swell will not be able to distinguish between these theories. The data should, however, be able to test the $k^{2} a^{3}$ dependence. However, the two mechanisms do differ in the transition to turbulent flow. For the Babanin (2012) theory, turbulent flow in the water occurs for Reynolds number $R_{e_{\text {wave }}}=a^{2} \omega / \nu_{w}>3 \times 10^{3}$, whereas for the Ardhuin et al. (2009) theory, turbulent flow in the air occurs for Reynolds numbers $R_{e}=8 a^{2} \omega / \nu_{a}>1 \times 10^{5}$. Note that in the Reynolds number formulation for Ardhuin et al. (2009), it has been assumed that $a_{\text {orb }}=\sqrt{2} a$, where $a$ is calculated for a monochromatic wave and $a_{\text {orb }}$ is determined from the significant wave height. If, as an example, we assume that $a=1 \mathrm{~m}, T=13 \mathrm{~s}, \nu_{a}=1.4 \times 10^{-5} \mathrm{~m}^{2} \mathrm{~s}^{-1}$, and $\nu_{w}=1.0 \times 10^{-6} \mathrm{~m}^{2} \mathrm{~s}^{-1}$, then for the Babanin (2012) theory $R_{e_{\text {wave }}}=4.8 \times 10^{5} \gg 3 \times 10^{3}$ and for the Ardhuin et al. (2009) theory $R_{e}=2.7 \times 10^{5}>1 \times 10^{5}$. In both cases, the flow will be turbulent. However, the Babanin (2012) theory (water) predicts an earlier transition to turbulence than the Ardhuin et al. (2009) theory (air).

The difference in transition from laminar to turbulent flow may provide a mechanism for determining which of the theories dominates. However, even for the Ardhuin et al. (2009) theory, laminar flow will only occur for small swell amplitudes. In these cases, measuring the small decay rates will be difficult, and hence, it is unlikely it would be possible to distinguish been the mechanisms in the data scatter. 

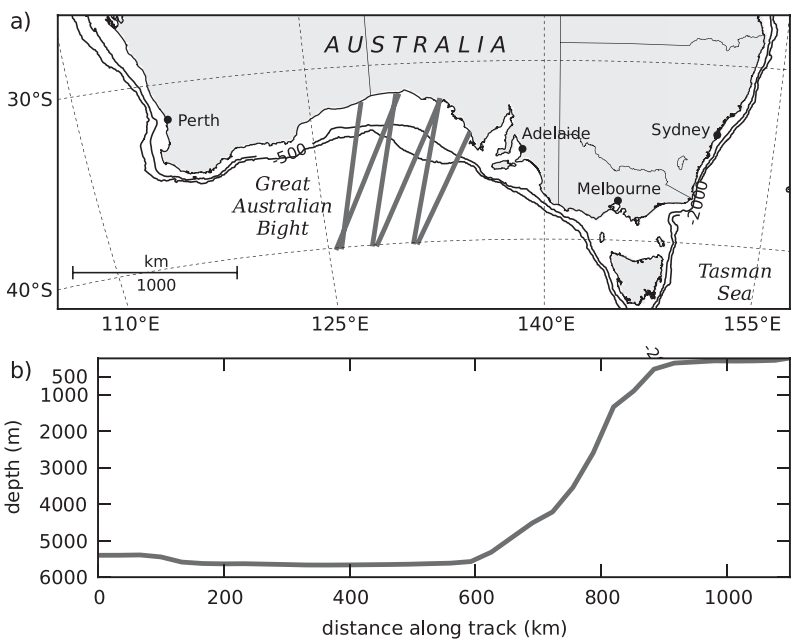

FIG. 1. (a) The study area in the Great Australian Bight region of the Southern Ocean. Typical altimeter ground tracks are shown across the region. (b) Variation of water depth along a typical altimeter great circle path. The point $x=0$ corresponds to the start of the track at approximately $40^{\circ} \mathrm{S}$.

Finally, it should be noted that the existing field datasets (Snodgrass et al. 1966; Collard et al. 2009) are quite sparse. Each experiment consists of only a few cases, the decay of which is measured at a limited number of locations.

\section{Altimeter database and study location}

For the present analysis, the altimeter database of Zieger et al. (2009) has been adopted. This database includes global altimeter observations of both significant wave height (i.e., $H_{s}$ ) and wind speed (i.e., $U_{10}$ ). The data span the period 1985-2008 and include all altimeter missions during this period. Each of the satellite missions was calibrated against data buoys and cross validated against coincident altimeter missions. For the purposes of the present study, only data from the European Remote Sensing Satellite (ERS)-1 (August 1991-May 1996), ERS-2 (April 1995-September 2008), Environmental Satellite (Envisat; September 2002-November 2008), and Ocean Topography Experiment (TOPEX) (September 1992-October 2005) missions were selected. Further missions could have been included, however, the selected missions provide extensive cases to consider and further data would add little additional information.

From the database, descending altimeter passes (i.e., from north to south) within the region bounded by latitudes $40^{\circ}-31.5^{\circ} \mathrm{S}$ and longitudes $124^{\circ}-136^{\circ} \mathrm{E}$ were extracted. This region is shown in Fig. 1a and roughly corresponds to the Great Australian Bight. This general area has been the subject of previous studies of bottom friction decay (closer to the coast) by Young and Gorman (1995). The region was selected as the descending altimeter passes closely conform to the direction of swell propagation in the region (Young and Gorman 1995). Figure $1 \mathrm{~b}$ shows the bathymetry along a sample altimeter pass from $40^{\circ} \mathrm{S}$ to the coast line. The propagation distance is approximately $1400 \mathrm{~km}$, the vast majority $(1200 \mathrm{~km})$ being in water depths greater than $500 \mathrm{~m}$ (i.e., deep water).

The buoy data recorded in the area by Young and Gorman (1995) show that the region is ideally situated to study swell propagation. The strong wind belts south of $40^{\circ} \mathrm{S}$ generate, year round, swell conditions propagating approximately from the south-southwest to the north-northeast. With the exception of periods when strong low pressure systems pass through the bight, conditions in the region are dominated by relatively homogeneous swell with $f<0.08 \mathrm{~Hz}$ (i.e., $T>12 \mathrm{~s}$ ).

Figures $2 \mathrm{a}$ and $2 \mathrm{~b}$ show typical situations in the Southern Ocean, as predicted by the WaveWatch model, version 3 (WaveWatch-III), with observationbased physics driven by Climate Forecast System Reanalysis, version 2 (CFSR-2), winds (Chawla et al. 2013; Zieger et al. 2011). The cases shown are for 16 June 2006 at 0600 UTC (Fig. 2a) and 14 October 2006 at 0000 UTC (Fig. 2b). It is clear from these figures that the Southern Ocean is characterized by a broad belt of high winds at a latitude of approximately $50^{\circ} \mathrm{S}$. Embedded within this belt are a series of stronger low pressure systems. This explains the consistent swell conditions observed in the Great Australian Bight. In the case of Fig. 2a, there are six distinct storms propagating across the Southern Ocean. These storms are large in spatial extent with wave heights $H_{s}>6 \mathrm{~m}$ typically extending for as much as $30^{\circ}$ latitude for each storm. For Fig. 2b, five storm centers can be distinguished but the wave fields generated by these storms have "joined" to create a broad belt stretching almost half way around the globe $\left(130^{\circ}\right)$ with $H_{s}>6 \mathrm{~m}$. This is quite a different situation to that considered by Snodgrass et al. (1966) and Collard et al. (2009) who investigated swell radiating out from an almost point source or isolated storm. This difference will be discussed further in section 4 .

From the extensive altimeter database, we wish to select cases for which the swell propagation direction is aligned with the great circle ground track of the altimeter and the wind speed is low (i.e., no/little atmospheric input). The altimeter records values of $H_{s}$ and $U_{10}$, but no information on wave propagation direction or wavenumber. To obtain estimates of wave (swell) direction and wavenumber, numerical model results from WaveWatch reanalysis data were selected (Chawla et al. 2013; 

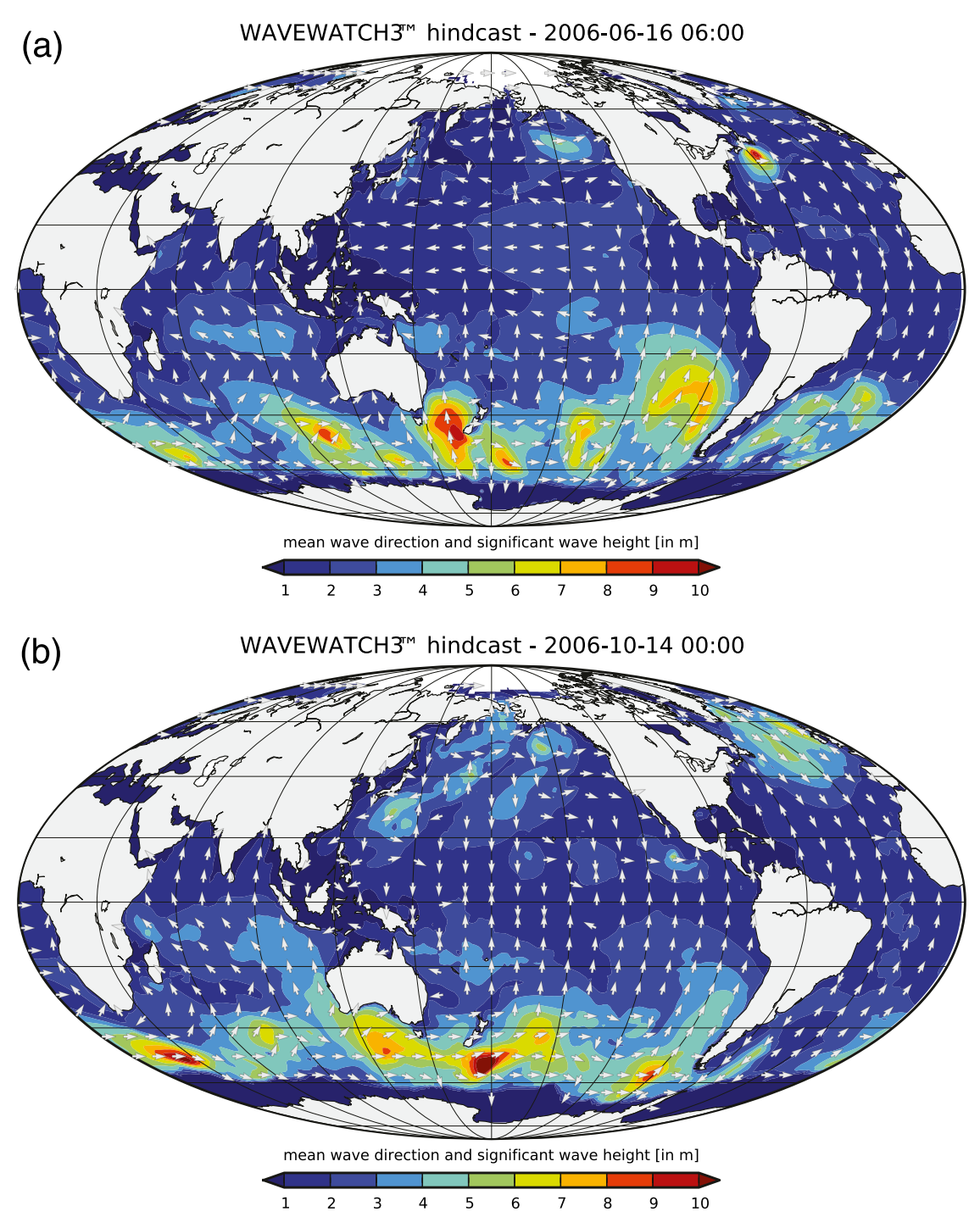

FIG. 2. Typical wave conditions in the Southern Ocean as predicted by the WaveWatch-III model. The cases shown are for (a) 16 Jun 20060600 UTC and (b) 14 Oct 20060000 UTC.

Tolman et al. 2011). Wave model data were selected at the closest grid points and times that corresponded to the most seaward points (i.e., approximately $40^{\circ} \mathrm{S}$ ) of each of the selected altimeter passes. A scatterplot comparing WaveWatch and altimeter $H_{s}$ is shown in Fig. 3. The WaveWatch wave height information is not directly used in this study. However, good agreement for $H_{s}$ provides confidence that the model will also produce reasonable estimates of swell direction and wavenumber. The agreement in Fig. 3 is reasonable, with the model tending to overestimate $H_{s}$ by approximately $0.5 \mathrm{~m}$. The Southern Ocean is a region with very limited meteorological data and hence wind field estimates and resulting wave predictions are often poor (e.g., Hemer et al. 2010). Noting this, the agreement in
Fig. 3 is reasonable, indicating that the model hindcasts are of good quality.

With this additional numerical model data, cases were selected where:

- Wave (swell) propagation direction, as predicted by the wave model, was within $\pm 10^{\circ}$ of the altimeter track direction at $40^{\circ} \mathrm{S}$. Values between $\pm 5^{\circ}$ and $\pm 15^{\circ}$ were tested and the results found to be insensitive to this variation.

- The wind speed, as measured by the altimeter, at all points along the altimeter track $\left(40^{\circ}-31.5^{\circ} \mathrm{S}\right)$ was less than $10 \mathrm{~m} \mathrm{~s}^{-1}$. Note that in all cases, the peak wave frequency, as predicted by the wave model, was less than $f=0.08 \mathrm{~Hz}$, for which $C>19 \mathrm{~m} \mathrm{~s}^{-1}$ and hence 


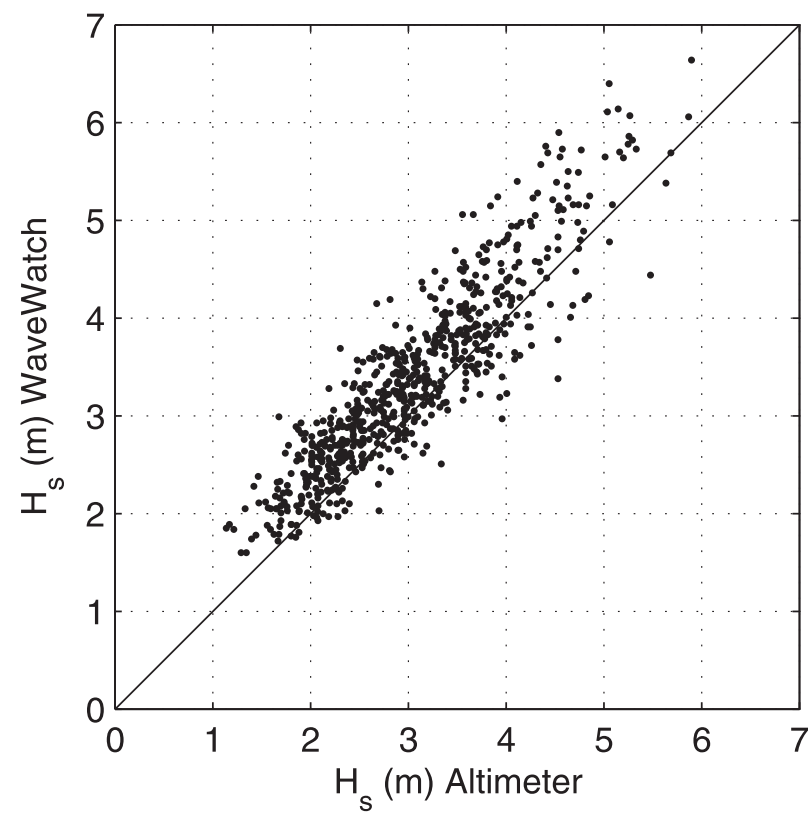

FIG. 3. Scatterplot comparing altimeter and WaveWatch-III estimates of the significant wave height (i.e., $H_{s}$ ). Comparisons are presented for each of 212 transects considered. The comparisons are presented at the "origin" point for each transect at approximately $40^{\circ} \mathrm{S}$.

$U_{10} / C<0.52$. That is, no/little wind input would be expected.

A total of 212 altimeter passes that satisfied these conditions were extracted from the database. An individual observation of $H_{\mathrm{ss}}$ (to determine the swell decay) is available every $1 \mathrm{~s}$ or approximately $7-8 \mathrm{~km}$ along each of these altimeter tracks.

Ideally, the aim would be to follow an individual swell train along the great circle path and monitor its passage. In the present case, however, the altimeter data are a nearly instantaneous transect of wave height along the great circle path. These two cases would be equivalent if the wave field in the area is approximately homogeneous over the period it takes for the swell to traverse the nearly $1000 \mathrm{~km}$ of the study area. For $f=0.08 \mathrm{~Hz}$ waves, the energy advection speed $C_{g}=g / 4 \pi f=9.75 \mathrm{~m} \mathrm{~s}^{-1}$. That is, it will take such waves some $29 \mathrm{~h}$ to traverse the area.

To test whether this is a reasonable assumption, the data of Young and Gorman (1995) were considered to understand the typical wave climate in the region. Data from station S1, the most seaward point of the Young and Gorman (1995) transect, were used. This station consists of buoy data recorded at a latitude of $34^{\circ} 24^{\prime} \mathrm{S}$ and $1150-\mathrm{m}$ water depth. Figure 4 shows a plot of the recorded buoy $H_{s}$ as a function of time for AugustOctober 1984. Data were generally recorded at an 8-h interval and this time series is plotted in Fig. 4. A 30-h window was passed over the data (i.e., the approximate propagation time for the swell along the altimeter ground track for the present study). Within this window, the minimum and maximum values of $H_{s}$ at S1 were recorded and these (the span from minimum to maximum) are shown as vertical bars in Fig. 4. For the assumption that the wave field is homogeneous over this propagation period to be true, this variability should be small. It should be noted that the stated accuracy of altimeter measurements of $H_{s}$ is $10 \%$ or $0.5 \mathrm{~m}$ (Monaldo 1988; Carter et al. 1992), whichever is the larger. The value $0.5 \mathrm{~m}$ is also shown in Fig. 4 as a reference. As can be seen, the variability in wave height over the 30-h period is of similar magnitude to the measurement accuracy of the altimeter. It should be noted that periods when the wind speed was greater than $10 \mathrm{~m} \mathrm{~s}^{-1}$ exhibited greater variability but such periods have not been considered in the present analysis. Therefore, it can be concluded that the assumption of homogeneity of the wave field on the scale of the propagation time is reasonable.

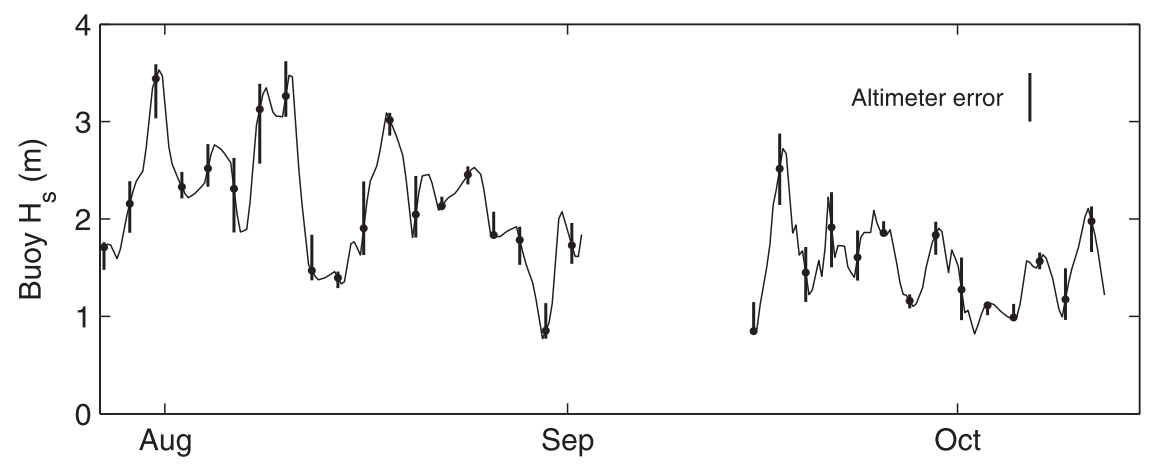

FIG. 4. The recorded buoy significant wave height (i.e., $H_{s}$ ) from location S1 of Young and Gorman (1995) at latitude $34^{\circ} 24^{\prime} \mathrm{S}$. The vertical lines represent the span (from min to max) for a 30 -h window across the data. Also shown is the typical altimeter measurement error $(0.5 \mathrm{~m})$. 


\section{Wave energy propagation model}

We aim to investigate the evolution of the wave energy with time (distance) along the one-dimensional altimeter great circle track. One could investigate this by running a full global wave prediction model such as WaveWatch-III (Tolman 2009) and studying the swell decay source term required to model the observed altimeter data. Although this appears an attractive approach, it has the limitation that such models have many other processes active in such situations. In addition to other source terms representing physical processes (many of which could be turned off, as shown below), the influences of numerical dispersion associated with the chosen propagation scheme will influence results. Although such effects must be considered when "tuning" source terms for use in such models, we aim to investigate the swell decay in isolation from such influences.

Collard et al. (2009) considered a similar problem and developed an approach to advect energy along a great circle path on the spherical surface of the Earth. In such a case, it is necessary to consider the effects of spherical spreading as a result of propagation over the earth's surface. A simple way to understand the process of spherical spreading is to consider the idealized case of a point generation source located at the South Pole (ignore land surfaces). Assume the propagation direction of the wave energy from this point source is north. Therefore, energy will propagate north along great circle paths which, in this case, are lines of longitude. Between the South Pole and the equator, the lines of longitude diverge in space (i.e., physical distance between the lines increases). Hence, the swell fronts will be stretched, resulting in a reduction in wave height, even in the absence of a physical process for swell decay. As shown by Collard et al. (2009) and Ardhuin et al. (2009), the reduction in energy for a spherical Earth can be calculated as

$$
\frac{E_{1}}{E_{2}}=\frac{\cos \phi_{2}}{\cos \phi_{1}}
$$

where $E_{1}$ and $E_{2}$ refer to the swell energy at points 1 and 2, which are at latitudes $\phi_{1}$ and $\phi_{2}$, respectively.

If the generation point source is not at the South Pole, (9) still applies but with the pole assumed to be located at the generation source and with angular distances $\phi$ measured from this source.

With distance from the point generation source, the ratio in (9) decreases. Similarly, if the generation source is not a point source, the effects of the stretching described by (9) will decrease. As shown in Fig. 2, the present situation is poorly represented by a point source and, hence, (9) would be an overestimate of the spherical spreading. As an order of magnitude estimate of the potential significance of this effect in the present case, it is assumed that the generation source is at the South Pole. In this case, the points at the most southern and northern points of the altimeter transect can be considered as points 1 and 2. That is $\phi_{1}=32^{\circ}$ and $\phi_{2}=40^{\circ}$ and the ratio $E_{1} / E_{2}=0.90$ or, if expressed in wave height, $H_{s_{1}} / H_{S_{2}}=\sqrt{0.90}=0.95$. That is, there would be a $5 \%$ reduction owing to spherical spreading over the measurement transect. Although this is only an illustrative example, combined with the situation shown in Fig. 2, it has been concluded that, in this case, spherical spreading will not be significant and can be ignored.

Noting the comments above, the advection of the directional spectrum in such a case can be written as (Komen et al. 1984; Young 1988; Komen et al. 1996)

$$
\frac{d}{d t}\left[C C_{g} F(f, \theta)\right]=C C_{g} S(f, \theta),
$$

where $C$ and $C_{g}$ are the phase speed and group velocity, respectively; and $S(f, \theta)$ is a source term that, in the present case, will consider the processes of swell decay and bottom friction decay (i.e., $S=S_{\mathrm{dec}}+S_{\mathrm{bf}}$ ). Other sources term processes are assumed to be small enough to neglect. Atmospheric input will be small as $U_{10} / C<1$. Because of the selection of low wind cases, nonlinear interactions will be small. White capping is small because the swell is largely monochromatic and there is no active wave breaking; depth-limited breaking can be ignored as water depths less than $10 \mathrm{~m}$ have been excluded from the calculations.

In the absence of more detailed information on the spectrum, and because the wind speed is relatively low, it is assumed that the spectrum is composed of a single swell component. Integrating (10) with respect to $f$ and $\theta$, noting that $H_{\mathrm{ss}}=2 a$, and converting (10) to a spatial derivative using $d / d t=C_{g} d / d x$ yields

$$
\begin{aligned}
C_{g} \frac{d}{d x}\left(\frac{C C_{g} H_{\mathrm{ss}}^{2}}{16}\right) & =C C_{g} \int S(f, \theta) d f d \theta \\
& =C C_{g}\left(I_{\mathrm{dec}}+I_{\mathrm{bf}}\right)
\end{aligned}
$$

where $I_{\mathrm{dec}}$ and $I_{\mathrm{bf}}$ are the integral forms of the swell decay and bottom friction source terms, respectively (see below).

Initially, it is assumed that the swell decay $I_{\mathrm{dec}}$ can be represented by the Babanin (2012) form (4), although it is noted that the physical process represented here could also be that of Ardhuin et al. (2009) and (5). In terms of $H_{\mathrm{ss}}$, (4) can be expressed as 


$$
C_{g} \frac{d}{d x}\left(\frac{H_{\mathrm{ss}}^{2}}{16}\right)=I_{\mathrm{dec}}=-\frac{C_{g}}{24} b_{1} k^{2} H_{\mathrm{ss}}^{3} .
$$

There are numerous forms that could be used for the bottom friction term, the most commonly used being Hasselmann and Collins (1968), Hasselmann et al. (1973), and WAMDI Group (1988). As noted earlier, Young and Gorman (1995) have already investigated bottom friction in this region and found that all forms can adequately model the decay, but with the Joint North Sea Wave Project (JONSWAP) form (Hasselmann et al. 1973; WAMDI Group 1988) yielding the best fit to recorded buoy data. The resulting source term becomes

$$
I_{\mathrm{bf}}=\frac{-\Gamma \omega^{2}}{16 g^{2} \sinh ^{2} k d} H_{\mathrm{ss}}^{2},
$$

where the decay coefficient varies with bed form (e.g., Mirfenderesk and Young 2003), but for this region could be approximated by the constant value $\Gamma=0.152 \mathrm{~m}^{2} \mathrm{~s}^{-3}$ (Young and Gorman 1995). Calculations ultimately show that the bottom friction term is small compared to the swell decay in water depths greater than $100 \mathrm{~m}$.

The differential equation, (11), was solved using a simple first-order forward difference scheme, in which the great circle altimeter track was discretized into spatial steps of $\Delta x \approx 100 \mathrm{~m}$, yielding the relationship

$$
H_{\mathrm{ss}_{i+1}}^{2}=\frac{C_{i} C_{g_{i}}}{C_{i+1} C_{g_{i+1}}} H_{\mathrm{ss}_{i}}^{2}+\frac{16 C_{i} I_{i} \Delta x}{C_{i+1} C_{g_{i+1}}},
$$

where the subscripts $i$ and $i+1$ refer to locations $x_{i}$ and $x_{i+1}$ along the great circle path, respectively, and the $C C_{g}$ ratio in the first term of (14) accounts for shoaling in finite depths. It should be noted that, as (14) is one dimensional, it does not account for any effects of refraction. However, the depth contours in the region have very limited curvature (it is a very long straight coastline and the satellite track is approximately shore normal). Therefore, refraction effects will be small. The subsequent analysis shows that the finite depth terms (bottom friction, shoaling, and refraction) are active over a relatively short part of the propagation distance (see Fig. 1) and hence have little impact on the analysis.

\section{Results and discussion}

The propagation model was applied to each of the 212 altimeter passes. Figure 5 shows typical examples of the comparison between the model results and the observed altimeter transect. The model contains only one unknown (or "tuneable" coefficient) $b_{1}$. A least squares fit to the cases gave the result $b_{1} \approx 0.0014$.

As can be seen in Fig. 5, the relationship (4) approximates the shape of the decay curve well, indicating that this functional form is a good fit to the data. This also supports the assumption that the flow (whether in the air or water or both) is turbulent and hence the functional form investigated is appropriate. The effects of bottom friction are only significant close to shore and can be seen by the discontinuity in each of the curves at the most northern point of the respective transects. If the finite depth component of the propagation path is neglected, a much simpler presentation of the data is possible, in terms of the nondimensional relationship (8). Figure 6 shows a plot of $H_{0} / H_{\mathrm{ss}}$ versus $H_{0} k^{2} x$, where $H_{0}$ is taken as the most seaward (southern) point on the great circle path, and $H_{\mathrm{ss}}$ is the observed value of swell along the path. Data from all 212 altimeter passes are shown. Only data for which $d>500 \mathrm{~m}$ have been included. The result for $b_{1}=0.0014$ is drawn as a straight line through the data. This relationship is clearly consistent with the data, although there is significant scatter. Such scatter is not surprising, noting the assumption that the swell field is homogeneous over the period of propagation, the measurement accuracy of the altimeter $\left[H_{s}-( \pm 0.5 \mathrm{~m})\right]$ and the potential influence of local wind and other wave systems contaminating the data.

It should be noted that (8) assumes that the flow is turbulent and that both the theories of Ardhuin et al. (2009) and Babanin (2011) have a critical Reynolds number that the flow (either in the air or water) must exceed before this turbulence decay mechanism can be active. Therefore, at small values of wave height this mechanism may cease to be effective. Order of magnitude estimates show this is not the case for the present dataset. Note that in any scenario, interaction of wave motion (even potential motion) with background oceanic turbulence on the water side should persist (e.g., Benilov 2012).

The results shown in Figs. 5 and 6 are clearly consistent with a relationship such as that proposed in (4), with the decay coefficient $b_{1} \approx 0.0014$. This value of $b_{1}$ can be compared with the previous field measurements of Snodgrass et al. (1966) and Collard et al. (2009). In each case, a comparison has been made at $x=5000 \mathrm{~km}$. The value $H_{0} / H_{\mathrm{ss}}$ can be calculated from (1) with the reported values of $\mu$. To give an equivalent $b_{1},(8)$ can then be solved. For Snodgrass et al. (1966), $T=13 \mathrm{~s}, k=$ $0.024 \mathrm{~m}^{-1}, H_{0}$ is assumed as $1.5 \mathrm{~m}$, and $\mu=2 \times 10^{-7} \mathrm{~m}^{-1}$. This gives a value $b_{1}=0.0014$, identical to the present experiment. For Collard et al. (2009), $T=15 \mathrm{~s}, k=$ $0.0179 \mathrm{~m}^{-1}, H_{0}=4.4 \mathrm{~m}$, and $\mu=3.7 \times 10^{-7} \mathrm{~m}^{-1}$. This gives a value of $b_{1}=0.0007$-half the value reported in 

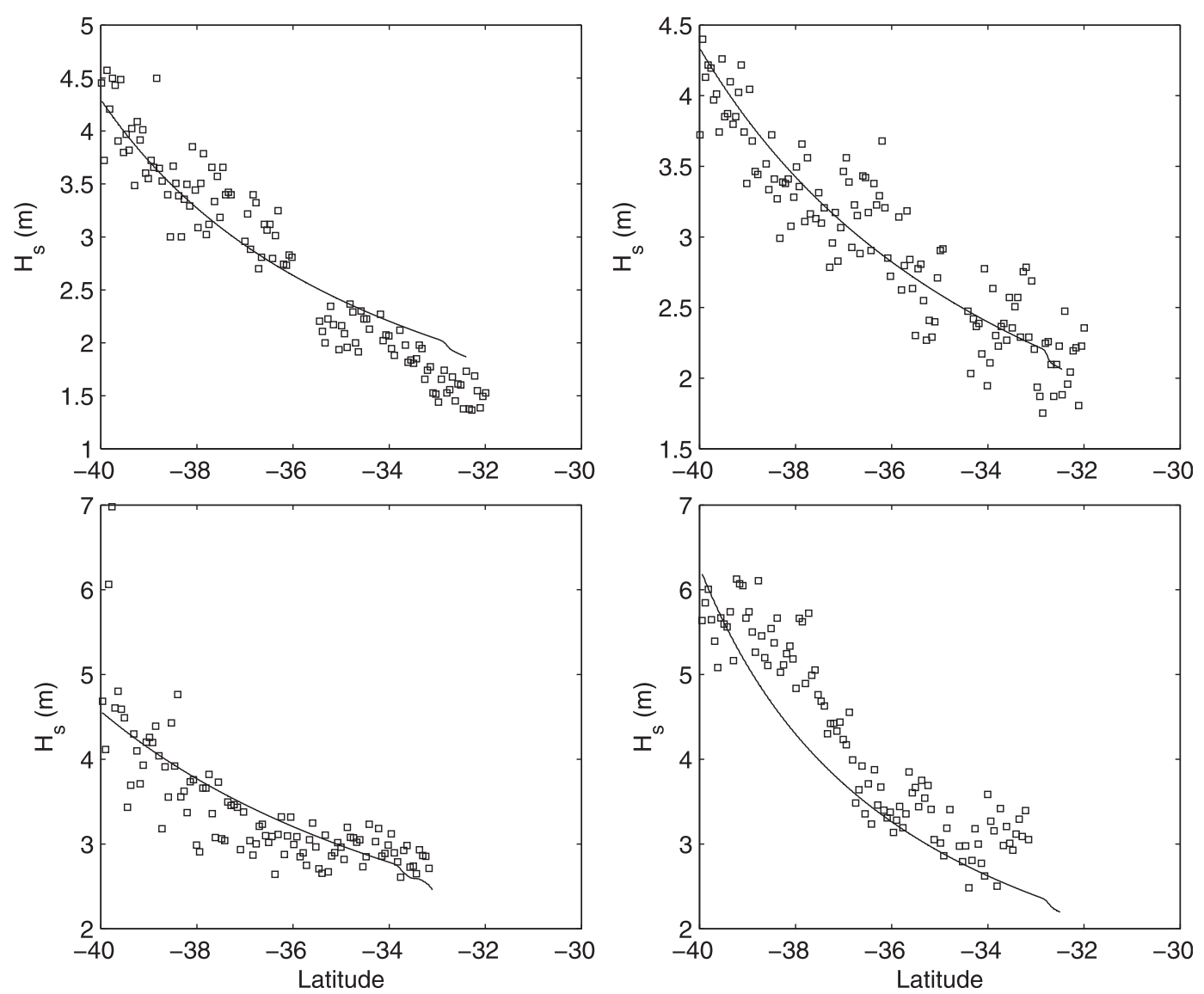

FIG. 5. Four typical cases of the measured (altimeter) decay of the swell along the transect (squares). The predicted decay from the model (14) is shown by the solid line.

the present experiment. This variability is, however, consistent with the experimental error in both studies. The value $b_{1} \approx 0.0014$ is consistent with these previous experiments. Estimates of Babanin (2012), as noted above, suggest a value of $b_{1}=0.001-0.004$, based on laboratory experiments. This, again, is consistent with the value obtained in the present study.

However, if one takes the relationship defined in (6), the value $b_{1}=0.0014$ can be converted to $f_{e}=0.058$, which is significantly larger than the range stated by Ardhuin et al. (2009) of $-0.001<f_{e}<0.019$. The comparisons above ignore the effects of spherical spreading approximated above by (9) for the case of a point source. If one ignores such effects then the present results are consistent with the measurements of Ardhuin et al. $(2009,2010)$. This then raises the question of whether the point assumption of (9) is reasonable. In the present case, we argue that this is certainly not the case and the generation source is a large region in many cases occupying a large fraction of the Southern Ocean. However, there will be some influence owing to propagation on the spherical surface of the earth. Thus, the value of $b_{1}=0.0014$ may represent an upper bound. One also must question, however, whether the point source assumption of Ardhuin et al. $(2009,2010)$ is completely valid, even for the cases reported by Collard et al. (2009). Therefore, it is possible that the Ardhuin et al. (2009) value of $f_{e}$ may represent a lower bound. If one takes the most common value of $f_{e}=0.007$ stated by Ardhuin et al. (2009), one obtains $b_{1}=0.00017$ from (6). Hence, the bounds on the coefficient appear to be $1.7 \times 10^{-4}<b_{1}<14.0 \times 10^{-4}$ or $7.0 \times 10^{-3}<f_{e}<$ $58.0 \times 10^{-3}$.

The results of the present experiment give good confirmation of the functional form for swell decay, as represented by (4) or (5), and the bounds on the decay coefficient. However, the ultimate test is to incorporate such a term in a full spectral model. In such a situation, the ultimate choice of the decay coefficient will be a "tuning" exercise with the range above providing order of magnitude guidance. This is a process outside the scope of the present paper. 


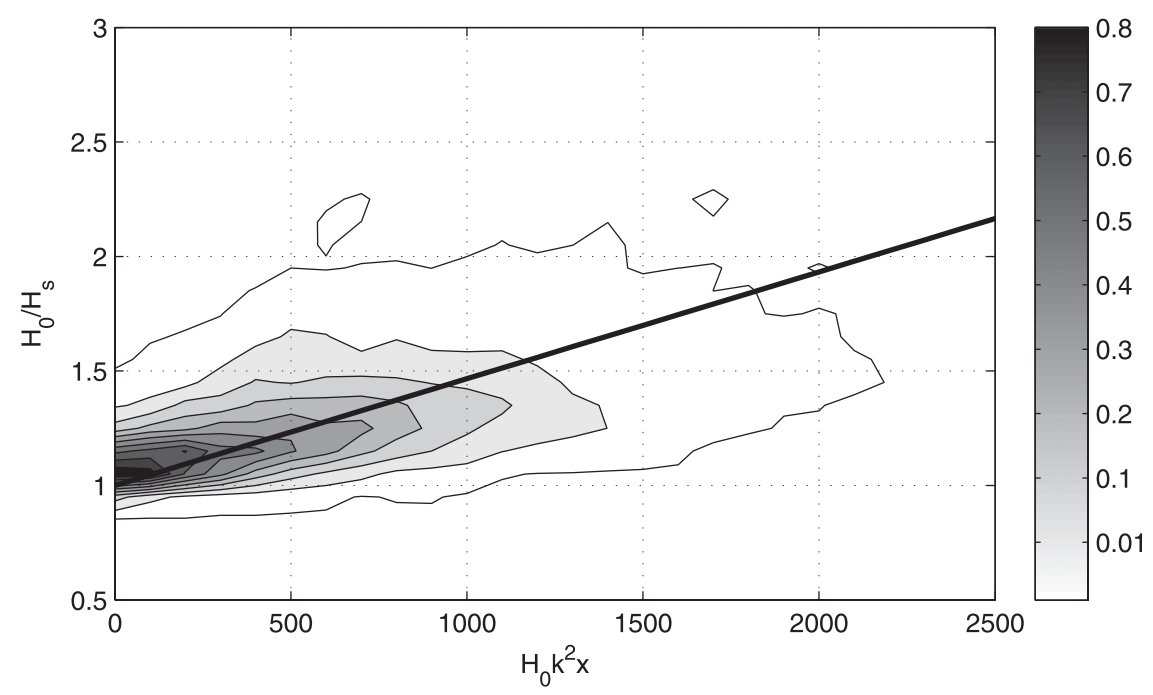

FIG. 6. Nondimensional decay as represented by (8) for all recorded altimeter measurements (212 cases). The solid line is for $b_{1}=0.0014$. The data consist of more than 23000 individual data points. The data density is contoured with a max contour value of 1 and contours drawn at $0.01,0.1,0.2,0.3,0.4,0.5,0.6,0.7$, and 0.8 .

However, the functional form of the source term which might be used in a spectral model can be tested against the observed decay rates from the present data. As the turbulent decay mechanisms described above are applicable to narrow-banded swell, some approximation is required to recast this in a spectral form.

The form proposed by Ardhuin et al. (2010) is

$$
S(f, \theta)=\frac{\rho_{a}}{\rho_{w}}\left(\frac{16 f_{e} \omega^{2} u_{\mathrm{orb}}}{g}\right) F(f, \theta),
$$

where $S(f, \theta)$ is the source term, $\omega=2 \pi f$ is the frequency, and the orbital velocity $u_{\text {orb }}$ can be approximated by $u_{\text {orb }}=2\left[\omega^{2} F(f, \theta) d f d \theta\right]^{1 / 2}$.

Alternatively, using (6), (15) can be written as

$$
S(f, \theta)=\left(\frac{2}{3} \frac{b_{1} \omega^{2} u_{\text {orb }}}{g}\right) F(f, \theta) .
$$

This alternative form of the swell dissipation, (16), was applied in WaveWatch by utilizing the one-dimensional propagation test provided with the model (i.e., ultimate quickest propagation scheme). In this academic test, spectral evolution is modeled along a single line. The spatial resolution was set at $100 \mathrm{~m}$ and the model was forced with swell applied at the boundary. This input swell condition was set as $H_{0}=4.5 \mathrm{~m}$ and a peak period of $T_{p}=14.9 \mathrm{~s}$. The swell was defined narrowly in both direction and frequency. The directional spread was defined as $\cos ^{n} \theta$, with $n=12$. The frequency distribution was Gaussian with a standard deviation of $1 \times 10^{-4} \mathrm{~Hz}$.
Other physical processes such as wind input, wave breaking, and white-capping dissipation and nonlinear wave-wave interactions were explicitly turned off. The decay of swell height as a function of propagation distance is depicted in Fig. 7. The agreement between the swell decay rate (8) and the spectral representation of swell dissipation (16) is excellent (correlation coefficient $r=0.9998)$. Note that the result was insensitive to the chosen boundary conditions, as long as it approximated a swell condition.

The empirical decay rate $\mu$, historically used by Snodgrass et al. (1966) and Collard et al. (2009), is also shown in Fig. 7 for $\mu=7 \times 10^{-7}$. Although this is a reasonable approximation to the observed shape of the decay curve, it should be noted that this is purely an empirical relationship with no physical underpinning.

\section{Conclusions}

As noted above, the importance of swell has been highlighted in recent times, with much of the global oceans dominated by such swell. Devising experiments to accurately measure the decay of swell over long distances is, however, challenging. Experiments that attempt to track individual swell trains across oceanic basins enable tight definition of the generation storm and swell system, but they inevitably result in a limited number of cases observed at a small number of locations. In contrast, the present paper investigates the decay of swell that propagates approximately along altimeter ground tracks. For such an analysis to be valid, the wave fields must be approximately homogeneous over the propagation time. 


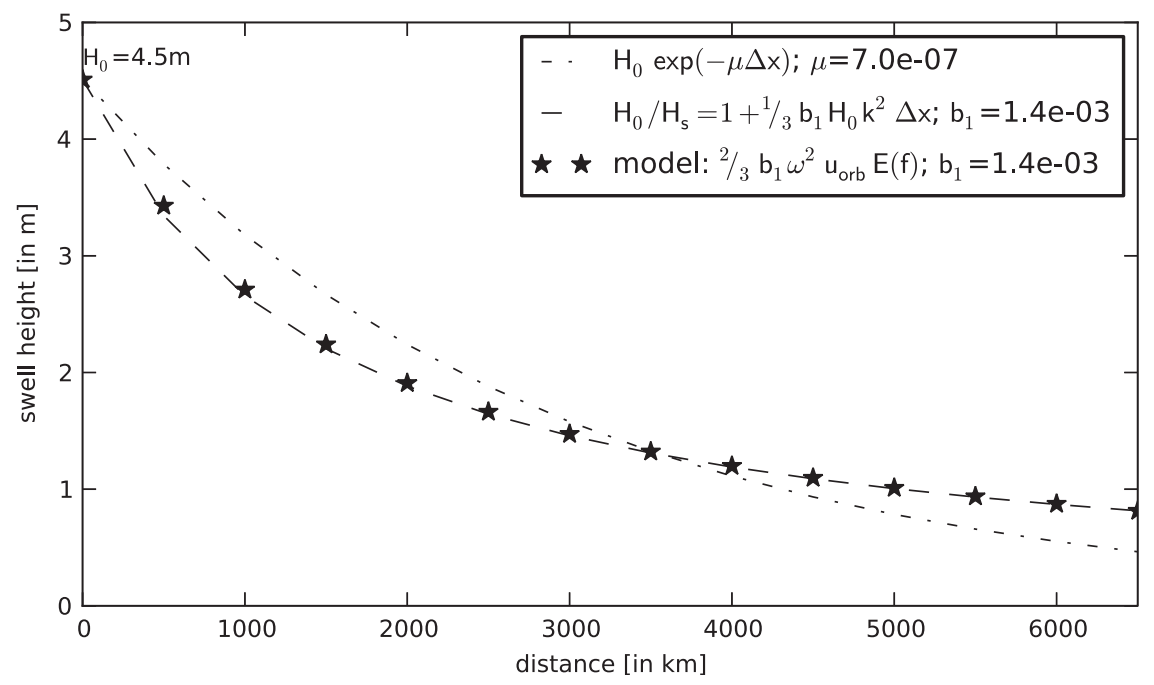

FIG. 7. Decay of swell in the spectral WaveWatch-III model for a test case with swell decay as the only active source term. The decay rate in the model using the source term (16) (stars) is compared with relationship (8) (dashed line) and the empirical decay relation (1) (dasheddotted line).

This limits both the geographic location and travel time for the experiment. Also, because of the limitation of the altimeter, the storms actually generating the swell cannot be defined. Therefore, the data are of lower quality than when tracking individual swell systems. However, as altimeter data are available for more than 23 years, many cases can be considered. Hence, a very extensive dataset covering a broad parameter range was investigated.

Data taken from the Southern Ocean that conforms to these strict selection criteria are considered (i.e., swell propagates approximately along the ground track, low wind, a region where the wave field is approximately homogeneous over $30 \mathrm{~h}$ ). The decay of such swell is found to approximate the functional forms proposed by both Ardhuin et al. (2009) and Babanin (2012) with a decay coefficient of $b_{1} \approx 0.0014$. Both of these previous studies assume swell decay to be the result of interaction with background turbulence. In the case of Ardhuin et al. (2009) the interaction is in the air, for Babanin (2012) it is within the ocean. As the functional forms of these theories are identical, the present study cannot separate the relative importance of each mechanism.

The observed decay is shown to be consistent with the limited previous field data. The resulting decay coefficient can be applied to ocean wave prediction models to account for swell decay. A detailed evaluation of the appropriate swell decay coefficient to use in a full spectral model is beyond the scope of this project. However, commonly used extrapolations of the observed swell decay relationships to spectral source terms have been shown to produce the same decay rates as the observations.
Acknowledgments. This work was financially supported by the Australian Research Council (ARC) through Linkage Grant LP0882422 and the U.S. Office of Naval Research (ONR) through Grant N00014-101-0418. The support of both the ARC and ONR and our industry partner RPS MetOcean is gratefully acknowledged.

\section{REFERENCES}

Ardhuin, F., B. Chapron, and F. Collard, 2009: Observation of swell dissipation across oceans. Geophys. Res. Lett., 36, L06607, doi:10.1029/2008GL037030.

— tions for ocean waves. Part I: Definition, calibration, and validation. J. Phys. Oceangr., 40, 1917-1941.

Babanin, A. V., 2006: On a wave-induced turbulence and a wavemixed upper ocean layer. Geophys. Res. Lett., 33, L20605, doi:10.1029/2006GL027308.

_- 2011: Breaking and Dissipation of Ocean Surface Waves. Cambridge University Press, $480 \mathrm{pp}$.

_ 2012: Swell attenuation due to wave-induced turbulence. Proc. 31st Int. Conf. On Ocean, Offshore and Arctic Eng., Rio de Janeiro, Brazil, OMAE2012, CD-ROM.

- and B. K. Haus, 2009: On the existence of water turbulence induced by non-breaking surface waves. J. Phys. Oceanogr., 39, 2675-2679.

Barstow, S. F., 1996: World wave atlas. AVISO Newsletter, No. 4, AVISO, Ramonville St-Agne, France, 24-35.

Benilov, A. Y., 2012: On the turbulence generated by the potential surface waves. J. Geophys. Res., 117, C00J30, doi:10.1029/ 2012JC007948.

Bowden, K. F., 1950: The effects of eddy viscosity on ocean waves. Philos. Mag., 41, 907-917.

Carter, D. J. T., P. G. Challenor, and M. A. Srokosz, 1992: An assessment of Geosat wave height and wind speed measurements. J. Geophys. Res., 97, 11383-11392. 
Chawla, A., D. M. Spindler, and H. L. Tolman, 2013: Validation of a thirty-year wave hindcast using the Climate Forecast System Reanalysis winds. Ocean Modell., 70, 189-206, doi:10.1016/j.ocemod.2012.07.005.

Chen, G., B. Chapron, and R. Ezraty, 2002: A global view of swell and wind sea climate in the ocean by satellite altimeter and scatterometer. J. Atmos. Oceanic Technol., 19, 1849-1859.

Collard, F., F. Ardhuin, and B. Chapron, 2009: Monitoring and analysis of ocean swell fields from space: New methods for routine observations. J. Geophys. Res., 114, C07023, doi:10.1029/2008JC005215.

Donelan, M. A., W. M. Drennan, and K. B. Katsaros, 1997: The air-sea momentum flux in conditions of wind sea and swell. J. Phys. Oceanogr., 27, 2087-2099.

Dore, B. D., 1978: Some effects of the air-water interface on gravity waves. Geophys. Astrophys. Fluid Dyn., 10, 215-230.

Gulev, S. K., and V. Grigorieva, 2006: Variability of the winter wind waves and swell in the North Atlantic and North Pacific as revealed by the voluntary observing ship data. J. Climate, 19, 5667-5685.

,--1, A. Sterl, and D. Woolf, 2003: Assessment of the reliability of wave observations from voluntary observing ships: Insights from the validation of a global wind wave climatology based on voluntary observing ship data. J. Geophys. Res., 108, 3236, doi:10.1029/2002JC001437.

Hasselmann, K., and J. I. Collins, 1968: Spectral dissipation of finite-depth gravity waves due to turbulent bottom friction. J. Mar. Res., 26, 1-12.

_ , and Coauthors, 1973: Measurements of wind-wave growth and swell decay during the Joint North Sea Wave Project. Dtsch. Hydrogr. Z., 8 (Suppl. A), 1- 95.

Hemer, M. A., J. A. Church, and J. R. Hunter, 2010: Variability and trends in the directional wave climate of the Southern Ocean Int. J. Climatol., 30, 475-491.

Hogstrom, U., A.-S. Smedman, E. Sahlee, W. M. Drennan, K. K. Kahma, C. Johansson, H. Pettersson, and F. Zhang, 2009: The atmospheric boundary layer during swell-A field study of the governing mechanism. J. Atmos. Sci., 66, 2764-2779.

Jensen, B. L., B. M. Sumer, and J. Fredsøe, 1989: Turbulent oscillatory boundary layers at high Reynolds numbers. J. Fluid Mech., 206, 265-297.

Komen, G. J., S. Hasselmann, and K. Hasselmann, 1984: On the existence of a fully developed wind sea spectrum. J. Phys. Oceanogr., 14, 1271-1284.

_ , L. Cavaleri, M. Donelan, K. Hasselmann, S. Hasselmann, and P. A. E. M. Janssen, 1996: Dynamics and Modelling of Ocean Waves. Cambridge University Press, 532 pp.

Lamb, H., 1932: Hydrodynamics. Cambridge University Press, 738 pp.
Mirfenderesk, H., and I. R. Young, 2003: Direct measurements of the bottom friction factor beneath surface gravity waves. Appl. Ocean Res., 25, 269-287.

Monaldo, F., 1988: Expected differences between buoy and radar altimeter estimates of wind speed and significant wave height and their implications on buoy-altimeter comparisons. J. Geophys. Res., 93, 2285-2302.

Semedo, A., O. Saetra, A. Rutgersson, K. Kahma, and H. Pettersson, 2009: Wave-induced wind in the marine boundary layer. $J$. Atmos. Sci., 66, 2256-2271.

- K. Suselj, A. Rutgersson, and A. Sterl, 2011: A global view on the wind sea and swell climate and variability from ERA-40. J. Climate, 24, 1461-1479.

Snodgrass, F. E., G. W. Groves, K. Hasselmann, G. R. Miller, W. H. Munk, and W. H. Powers, 1966: Propagation of ocean swell across the Pacific. Philos. Trans. Roy. Soc. London, A249, 431-497.

Sterl, A., and S. Caires, 2005: Climatology, variability and extrema of ocean waves: The Web-based KNMI/ERA-40 wave atlas. Int. J. Climatol., 25, 963-977, doi:10.1002/joc.1175.

Tolman, H. L., 2009: User manual and system documentation of WAVEWATCH III version 3.14. NCEP Tech. Note 276, $194 \mathrm{pp}$.

—, M. L. Banner, and J. M. Kaihatu, 2011: The NOPP operational wave model improvement project. Proc. 12th Int. Workshop on Wave Hindcasting and Forecasting, Kohala Coast, HI, WMO/IOC, CD-ROM.

WAMDI Group, 1988: The WAM Model-A third generation ocean wave prediction model. J. Phys. Oceanogr., 18, 17751810.

Young, I. R., 1988: A shallow water spectral wave model. J. Geophys. Res., 93 (C5), 5113-5129.

, 1999: Seasonal variability of the global ocean wind and wave climate. Int. J. Climatol., 19, 931-950.

— , and R. J. Sobey, 1985: Measurements of the wind-wave energy flux in an opposing wind. J. Fluid Mech., 151, 427-442.

_ , and R. M. Gorman, 1995: Measurements of the evolution of ocean wave spectra due to bottom friction. J. Geophys. Res., 100 (C6), 10 987-11004.

Zieger, S., J. Vinoth, and I. R. Young, 2009: Joint calibration of multi-platform altimeter measurements of wind speed and wave height over the past 20 years. J. Atmos. Oceanic Technol., 26, 2549-2564.

- A. Babanin, W. E. Rogers, and I. R. Young, 2011: Observationbased dissipation and input terms for a WAVEWATCH III implementation and simple simulations. Preprints, 12th Int. Workshop on Wave Hindcasting and Forecasting, Kohala Coast, HI, WMO/IOC, CD-ROM. 